\title{
Determination of Rare Earth Elements in Geological Samples by Inductively Coupled Plasma Atomic Emission Spectrometry with Flow Injection Liquid-Liquid Extraction
}

\author{
Zhifang XU, ${ }^{* \dagger}$ Congqiang LIU,** Hongxiang ZHANG,* Yingjun MA,* and Soulin LIN*** \\ *Institute of Geology and Geophysics, Chinese Academy of Sciences, Beijing 100029, \\ People's Republic of China \\ **Institute of Geochemistry, Chinese Academy of Sciences, Guiyang 550002, People's Republic of China \\ ***Department of Applied Chemistry, China University of Geosciences, Wuhan 430074, \\ People's Republic of China
}

\begin{abstract}
A direct sampling with organic solvent extracts for simultaneous multi-element determination implemented with inductively coupled plasma atomic emission spectrometry (ICP-AES) associated with a flow injection liquid-liquid extraction (FI-LLE) sample preconcentration method was studied. The "robustness" of the plasma discharge with tributyl phosphate (TBP) loading was diagnosed by using the Mg II $279.55 \mathrm{~nm}$ and Mg I $285.21 \mathrm{~nm}$ lines intensity ratio. A FILLE preconcentration system for rare earth elements (REEs)-nitrate-TBP was established by using a laboratory-designed phase separator. For these elements, an average sensitivity enhancement factor of 64 was obtained with respect to ICPAES sampling with aqueous solutions. The precision of the method was characterized by a relative standard deviation (\%RSD) of $1.8-5.2 \%$. A throughput of 27 samples per hour can be achieved with an organic solvent consumption of less than $200 \mu \mathrm{l}$ per determination. Good results were obtained for the analysis of standard reference materials.
\end{abstract}

(Received June 23, 2003; Accepted September 2, 2003)

\section{Introduction}

As one of the preferred methods for simultaneous multi-element determination, inductively coupled plasma atomic emission spectrometry (ICP-AES) has been widely applied to the determination of trace elements in various materials. ${ }^{1-5}$ Yet, its use in analyses of complex samples for elements at trace or ultra-trace levels is still limited, owing to insufficient sensitivity and matrix effects. ${ }^{6-8}$ Therefore, methods of ICP-AES associated with the separation and preconcentration procedures are needed. ${ }^{9}$ The solvent-extraction technique is an effective approach for the preconcentration of detecting elements and the separation of matrix elements; also, the presence of organic solvent may improve the nebulization and transported efficiencies.

However, the presence of organic solvents in the plasma has a profound effect on the plasma discharge characteristics. Solvent vaporization and dissociation processes absorb energy from the plasma, and therefore decrease the populations of the excited state level. Solvent-plasma interactions have been investigated for a number of years. For example, Boorn and Browner made a survey of fundamental analyte transport and solvent vaporization properties when organic solvents were used in conventional ICP-AES, and pointed out that some organic solvents might cause the destabilization or extinction of a low-power argon ICP discharge owing to the high relative solvent vapor pressure in the discharge and the solvent loading

† To whom correspondence should be addressed.

E-mail: zfxu@mail.igcas.ac.cn of the plasma. ${ }^{10}$ Boumans and Lux-Steiner described experiments aimed at the optimization of the operating conditions of the ICP system using methl isobutyl ketone (MIBK) as an organic solvent. ${ }^{11}$ Maessen et al. investigated the effects of chloroform loading on the analytical performance of ICP-AES. ${ }^{12}$ Pan et al. studied the influence of carbon tetrachloride on the ICP characteristics, and suggested that the main impact of desolvation with organic solvents is to reduce the $\mathrm{C}_{2}$ species population in the plasma. ${ }^{13}$ Weir and Blades provided a detailed description of the effect of added organic aerosols on the spectra and the appearance of ICP discharge, and studied the influence of solvent loading, the power and gas flow rate on ICP discharge and background emissions. ${ }^{14,15}$ In addition, the manual liquid-liquid extraction procedures are usually very time-consuming, the consumption of solvents and chemicals is high, and the volatility of solvents may cause the environmental contamination. The application of solvent extraction directly associated with ICP-AES to the routine analysis of real samples has been rarely reported.

A very effective way to overcome the above-mentioned drawbacks is an automated online liquid-liquid extraction technique. Since the appearance of flow injection (FI) analysis, much work involving flow injection liquid-liquid extraction (FILLE) has been reported. ${ }^{16-20}$ The phase separator is the most critical component of FI-LLE systems. We have studied the phase separator for several years. ${ }^{21,22}$ In this work, a laboratorydesigned glass gravitational phase separator is used. The separator has been proved to have the following advantages: 1, long-term stability, no dead volume and easy cleaning; 2 , usable for organic solvents either heavier or lighter than water; 3 , it can be connected with various detectors, such as atomic absorption 
Table 1 Instrumental conditions for FI-LLE-ICP-AES traceelement analysis

\begin{tabular}{ll}
\hline $\begin{array}{l}\text { Operating conditions for ICP-AES } \\
\text { Wavelength (nm) }\end{array}$ & La 333.75; Nd 309.42; Sm \\
& $359.26 ;$ Eu 381.97; Er 337.3; \\
& Yb 328.94; Y 324.23 \\
& 2.85 \\
Generator power $(\mathrm{kW})$ & 12 \\
Argon gas flow: & 0.33 \\
Plasma gas flow rate $(1 / \mathrm{min})$ & 1.0 \\
Auxiliary gas flow rate $(1 / \mathrm{min})$ & 12 (above the coil) \\
Carrier gas flow rate $(1 / \mathrm{min})$ & 0.60 \\
Observation height $(\mathrm{mm})$ & \\
Solvent uptake rate $(\mathrm{ml} / \mathrm{min})$ & \\
& \\
Operating conditions for the flow injection \\
Length of extraction coil (mm) & 2500 \\
Extraction coil diameter (mm) & 25 \\
Inner diameter of tubing (mm) & 0.9 \\
Injected volume of the organic & 200 \\
extract $(\mu \mathrm{l})$ &
\end{tabular}

spectrometers, spectrophotometers, and ICP-AES, etc.

In the present work, direct sampling with organic solvent extracts for simultaneous multi-element determination by the combination of ICP-AES and FI-LLE preconcentration method was studied. The operating parameters for ICP-AES and the factors that affect the FI-LLE preconcentration system were investigated in detail. It was found that plasma discharge operating with tributyl phosphate (TBP) loading was robust, if the operating parameters for ICP-AES and the solvent plasma loading were selected properly. By means of a laboratorydesigned separator, a flexible manifold for FI-LLE was designed and the analytical performance of FI-LLE-ICP-AES was evaluated. Studies based on a rare earth elements (REEs)nitrate-TBP system indicate that the combination of FI-LLE and ICP-AES is feasible and applicable.

\section{Experimental}

\section{Apparatus}

ICP source: Model G.P. 3.5-D1 (Xinxing Machine Factory, Shanghai, China); frequency, $30-40 \mathrm{MHz}$; and maximum power output, $3.5 \mathrm{~kW}$.

Optical system: Laboratory-made sequential spectrometer; a WPG-100 plane grating spectrograph (Beijing Second Optical Instrumrnt Factory, China) was used as a monochromator; $1 \mathrm{~m}$ mounting; 1200 grooves $/ \mathrm{mm}$ grating blazed at $300 \mathrm{~nm}$; and reciprocal dispersion, $0.8 \mathrm{~nm} / \mathrm{mm}$. The operating conditions for ICP-AES are listed in Table 1.

Flow injection system: IFIS-A intelligent flow injection sampler (Institute of Spring, Xian, China), with two threechannel peristaltic pumps and an eight-channel rotary injection valve. The flow injector was fully interfaced to the ICP-AES, and the operating conditions are also listed in Table 1.

\section{Reagents}

Magnesium-tributyl phosphate (Mg-TBP) solution (Mg 10 $\mu \mathrm{g} / \mathrm{ml}): 0.1 \mathrm{ml}$ magnesium standard solution $(2.5 \mathrm{mg} / \mathrm{ml})$ was transferred to a $25 \mathrm{ml}$ volumetric flask and diluted with TBP to $25 \mathrm{ml}$, then shaken well.

Standard solutions of rare-earth elements (REEs) $(1 \mathrm{mg} / \mathrm{ml})$ were prepared from their oxides. Calibration standards were prepared by successive dilution with water. Final multi-element
Table 2 Manifold program for FI

\begin{tabular}{|c|c|c|c|c|c|c|c|}
\hline & & \multicolumn{6}{|c|}{ Step } \\
\hline & & 1 & 2 & 3 & 4 & 5 & 6 \\
\hline \multirow[t]{2}{*}{ Pump A } & Time (s) & 20 & 40 & 5 & 30 & 5 & 30 \\
\hline & Speed $(r / \mathrm{min})$ & 60 & 4 & 0 & 0 & 0 & 8 \\
\hline \multirow[t]{2}{*}{ Pump B } & Time $(s)$ & 20 & 40 & 5 & 30 & 5 & 30 \\
\hline & Speed $(r / \min )$ & 0 & 67 & 0 & 70 & 0 & 0 \\
\hline Valve po & ition & a & $\mathrm{b}$ & & & & a \\
\hline \multicolumn{8}{|c|}{ Unit of pump speed: rotations per minute } \\
\hline \multicolumn{8}{|c|}{ Flow rate corresponding to pump speed is as follows: } \\
\hline \multicolumn{3}{|c|}{ Speed of pump A ( $r / \mathrm{min})$} & 12 & 10 & 8 & 6 & 4 \\
\hline \multicolumn{3}{|c|}{ Flow rate $(\mathrm{ml} / \mathrm{min})$} & 0.90 & 0.75 & 0.60 & 0.45 & 0.30 \\
\hline \multicolumn{3}{|c|}{ Speed of pump B $(r / \mathrm{min})$} & 80 & 70 & 60 & 50 & 40 \\
\hline \multicolumn{3}{|c|}{ Flow rate $(\mathrm{ml} / \mathrm{min})$} & 7.20 & 6.30 & 5.40 & 4.50 & 3.60 \\
\hline
\end{tabular}

Table 3 Time cycle of determinations

\begin{tabular}{|c|c|c|}
\hline Step & Time/s & Function \\
\hline 1 & $0-20$ & $\begin{array}{l}V \text { remains in position a. } P_{A} \text { activates. } P_{B} \text { stops. } \\
\text { The phase separator and ICP-AES is washed with } \\
\text { water. }\end{array}$ \\
\hline 2 & $21-60$ & $\begin{array}{l}\mathrm{V} \text { is turned to position } \mathrm{b} . \mathrm{P}_{\mathrm{A}} \text { and } \mathrm{P}_{\mathrm{B}} \text { activate. Start } \\
\text { the extraction process. }\end{array}$ \\
\hline 3 & 5 & $\begin{array}{l}P_{A} \text { and } P_{B} \text { stop. Sample solution is replaced by } \\
\text { water. }\end{array}$ \\
\hline 4 & 95 & $\begin{array}{l}P_{A} \text { stops. } P_{B} \text { activates. The segmented phases } \\
\text { remaining in the extraction coil are completely } \\
\text { carried into the phase separator. }\end{array}$ \\
\hline 5 & 00 & $\begin{array}{l}\mathrm{P}_{\mathrm{A}} \text { and } \mathrm{P}_{\mathrm{B}} \text { stop. The organic phase is completely } \\
\text { separated from aqueous phase in the phase } \\
\text { separator. }\end{array}$ \\
\hline 6 & $101-130$ & $\begin{array}{l}\mathrm{V} \text { is turned to position a. } \mathrm{P}_{\mathrm{A}} \text { activates. } \mathrm{P}_{\mathrm{B}} \text { stops. } \\
\text { The solvent extract is carried into ICP-AES by } \\
\text { water. }\end{array}$ \\
\hline
\end{tabular}

test solutions were acidified to $3 \%$ with nitric acid. All used reagents were at least of analytical reagent grade. Distilled water was used throughout.

\section{Manifolds and operating procedures}

The extraction manifold using an eight-channel valve and 0.9 $\mathrm{mm}$ inner diameter by $2.5 \mathrm{~m}$ long PTFE tubing as an extraction coil is shown in Fig. 1. A trisection confluence connector with a $0.9 \mathrm{~mm}$ inner diameter was used as a segment generator. The flow rates used were $6.0 \mathrm{ml} / \mathrm{min}$ for the sample and $0.30 \mathrm{ml} / \mathrm{min}$ for TBP. To facilitate the attainment of the desired flow-rate ratios, the two phases were propelled by separate peristaltic pumps. The sample and TBP passed the extraction coil, proceed to the segment and finally entered the phase separator. The TBP was automatically separated from the aqueous phase owing to the difference in the specific gravities of the two phases. The solvent extract was then propelled into the nebulizer of the spectrometer for an ICP-AES measurement. The manifold program and time cycle of the determination are given in Tables 2 and 3.

Procedure for REEs determination: After a certain volume of sample solution was pipetted into a $25 \mathrm{ml}$ volumetric flask, 14 $\mathrm{ml}$ of concentrated nitric acid was added, then cooled down and made up to volume with water and shaken well. Following the selected manifold and operating sequence, extraction with TBP was done and a determination with ICP-AES was made. 

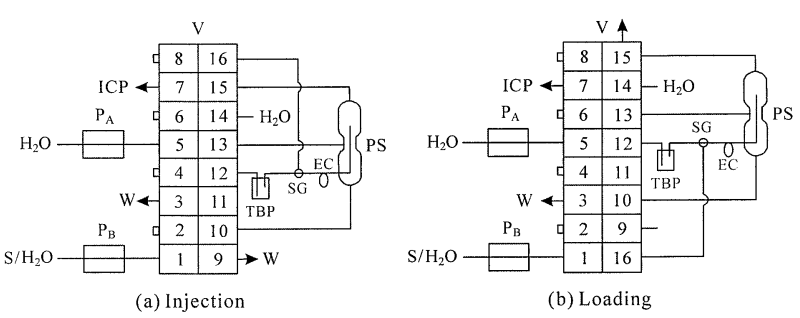

Fig. 1 Manifold for FI-LLE-ICP-AES. $\mathrm{P}_{\mathrm{A}}$ and $\mathrm{P}_{\mathrm{B}}$, three-channel peristaltic pump; V, eight-channel rotary valve; PS, phase separator; EC, extraction coil; SG, segment generator; TBP, tributyl phosphate; $\mathrm{S}$, sample solution; $\mathrm{W}$, waste.

\section{Results and Discussion}

\section{Optimization of the ICP operating conditions}

The introduction of an organic solvent into ICP requires optimization of the plasma operating conditions, including the forward power, carrier gas-flow rate, observation height and solvent plasma loading, since those conditions used for aqueous solution are not suitable. Because the decomposition of organic molecular compounds consumes more energy, the introduction of an organic solvent may obviously change the characteristics of the ICP discharge, or make it destabilize and extinguish. Diagnostics must be applied to the ICP to obtain an understanding of the basic processes that transform the injected species into photons or ions, and to optimize the operating parameters of the plasma.

One major criterion for understanding the plasma processes is the closeness to the local thermodynamic equilibrium (LTE). The ionic-to-atomic line intensity ratio allows a measurement of the departure from LTE and a verification of the optimization of the operating parameters of the plasma. The advantage of this method is its simplicity. Among the possible elements, magnesium $(\mathrm{Mg})$ has often been used. ${ }^{13,15,23}$ In particular, selection of the $\mathrm{Mg}$ I line at $285.21 \mathrm{~nm}$ and of the $\mathrm{Mg}$ II lines at $280.27 \mathrm{~nm}$ (or $279.55 \mathrm{~nm}$ ) is favored because of several practical advantages and physical attributes. Mermet summarizes many of these: 1 , the excitation potential of the $\mathrm{Mg}$ I $285.21 \mathrm{~nm}$ line is close to that of Mg II $280.27 \mathrm{~nm}$ and 279.55 $\mathrm{nm}$ lines, so that the effect of the second exponential term is negligible; 2, the two $\mathrm{Mg}$ II lines have been proved to be sensitive to the ICP excitation condition, so that a ratio incorporating one of them will also be a sensitive measure; 3 , the closeness of wavelengths of the atomic and ionic lines usually avoids the spectral response correction. In addition, the $\mathrm{Mg}$ ratios were favored because of the absence of molecular background emission features in the $\mathrm{Mg}$ II and $\mathrm{Mg}$ I wavelength region. ${ }^{23}$

In the experiment, a Mg-TBP solution in a displacement bottle was sampled by using a flow injection sampler; the manifold is shown in Fig. 2. First, the valve remains in position a, pump A and pump B activate, filling the sampling loop with Mg-TBP and washing the manifold with water. The valve is then turned to position $b$, pump A stops and pump B activates, carrying a certain amount of Mg-TBP into the ICP-AES, and the intensities of $\mathrm{Mg}$ I $285.21 \mathrm{~nm}$ and $\mathrm{Mg}$ II $279.55 \mathrm{~nm}$ lines are determined. Figure 3 is the parametric response of the line of $\mathrm{Mg}$ ion-to-atom line intensity ratio for ICP loaded with TBP. If one accepts Mermet's proposal that the Mg ratio approaches 20 for a robust plasma close to LTE, then the profiles indicate that LTE is only approached for low solvent loads $(<1.0 \mathrm{mg} / \mathrm{s})$, high power $(>2.85 \mathrm{~kW})$, a low carrier gas flow rate $(<1.00 \mathrm{l} / \mathrm{min})$ and

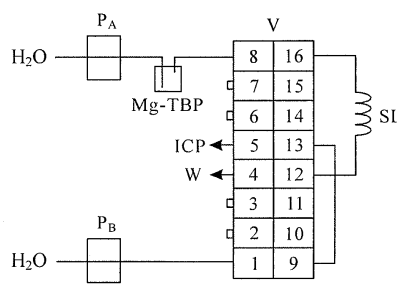

(a) Loading

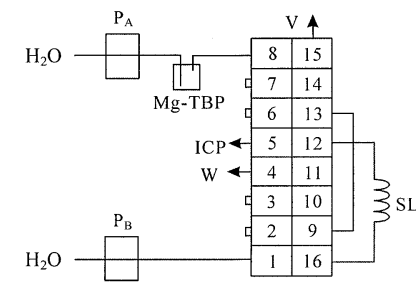

(b) Injection
Fig. 2 Manifold for diagnosing the plasma discharge. $P_{A}$ and $P_{B}$, three-channel peristaltic pump; V, eight-channel rotary valve; SL, sampling loop (Teflon, $100 \mathrm{~mm}$ length, i.d. $0.9 \mathrm{~mm}$ ); W, waste.

a proper observation height $(10-16 \mathrm{~mm}$ above the coil). From a diagnosis of the ICP discharge, it can be concluded that the plasma appears to approach LTE with TBP loading under these conditions, and corresponds to an optimization of the ICP discharge.

\section{Optimization of the manifold parameters}

Some manifold parameters, including the Teflon tube diameter, extraction coil diameter, and coil length, should be optimized in the application of FI-LLE. Experiments are performed by changing one parameter at a time while other parameters remain fixed. It can be seen from Figs. 4, 5 and 6 that the optimized conditions are: Teflon tube inner diameter, $0.9 \mathrm{~mm}$; extraction coil diameter, $25 \mathrm{~mm}$; and coil length, 2.5 $\mathrm{m}$. It is found that both the tube diameter and the coil diameter are critical factors affecting the extraction efficiency.

\section{Influence of the phase flow ratio}

The concentration factor value depends on the flow ratio of the aqueous phase to the organic phase, and it would seem that the greater is the ratio, the greater will be the concentration factor. However, this is not necessarily always true, and there are practical limits to the ratio. It can be seen from Fig. 7 that the curve approaches a maximum plateau at a point corresponding to a flow ratio of 20 . A very high phase flow ratio and total flow rate are not preferred. A very high phase flow ratio and total flow rate may cause a loss of the extraction efficiency. Hysteresis of an organic solvent in the extraction coil was observed. It was found that TBP can not be completely driven out of the extraction coil by water.

\section{Injected volume of the solvent extract}

The injected volume of the solvent extracts needs to reach the equilibrium signal for ICP-AES. The experimental results are plotted in Fig. 8. In the flow system used in this work for the determination of REEs, an injected volume of $200 \mu \mathrm{l}$ was used.

\section{Analytical performance of FI-LLE-ICP-AES}

The analytical performance achieved by the proposed FI-LLEICP-AES system, including the detection limits, precision, sample throughout, sample consumption and TBP consumption, are listed in Table 4. The detection limits (DL) were calculated based on three-times the standard deviation of 7 measurements. In the experiment, an old laboratory-made ICP-AES system was used because of considering the effects of an organic solvent on the ICP, and thus the detection limits in aqueous solution were high. However, from a comparison between flow-injection solvent extraction and direct nebulization with an aqueous solution, it is clear that a great improvement in the detection limits has been obtained. The average sensitivity enhancement factor of the detection limits is 64 . The precisions were 

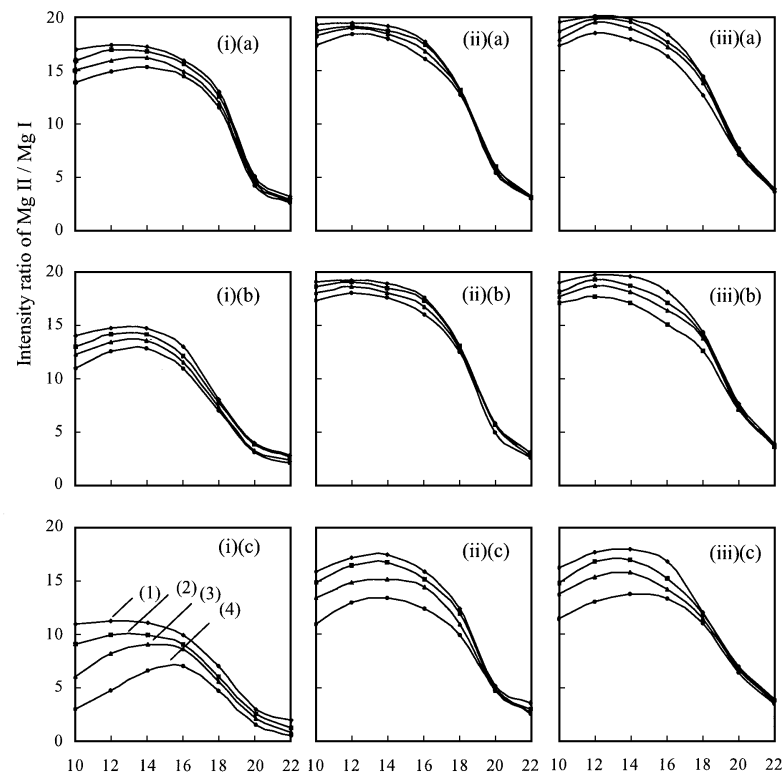

Observation height $/ \mathrm{mm}$

Fig. 3 Influence of the solvent plasma loading (SPL) and the operating parameters for ICP on the intensity ratio of Mg II 279.55 $\mathrm{nm}$ to $\mathrm{Mg}$ I $285.21 \mathrm{~nm}$. Power: (i) 2.7 , (ii) 2.85 , (iii) $3.0 \mathrm{~kW}$; TBP plasma loading (the amount of solvent delivered to the discharge per unit time): (a) 0.54 , (b) 0.82 , (c) $1.0 \mathrm{mg} / \mathrm{s}$; carrier gas-flow rate: (1) 0.67 , (2) 0.83 , (3) 1.00 , (4) $1.17 \mathrm{l} / \mathrm{min}$.

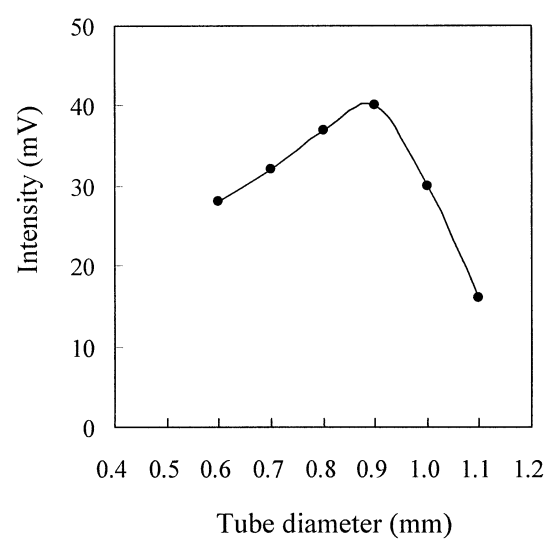

Fig. 4 Emission signal as a function of the tube inner diameter, concentration of $400 \mu \mathrm{g} / \mathrm{l}$ ytterbium.

evaluated using standard solutions of these elements, containing $0.4 \mu \mathrm{g} / \mathrm{ml}$ for FI-LLE and $10 \mu \mathrm{g} / \mathrm{ml}$ for direct nebulization with an aqueous solution, respectively. The values of the relative standard deviations (RSD \%) were calculated from 7 replicates, were often lower than $5.2 \%$ for FI-LLE-ICP-AES, and showed that the reproducibility was good. From the time cycle of the determination listed in Table 3, the overall time required for the preconcentration and detection of one sample was approximately $2.1 \mathrm{~min}$. Thus, the sample throughput was approximately 27 samples/h.

\section{Sample analysis}

In order to assess the feasibility of the combination of FI-LLE and ICP-AES, the proposed method was applied to the analysis of geological standard reference materials. The results are presented in Table 5. The values obtained for the reference

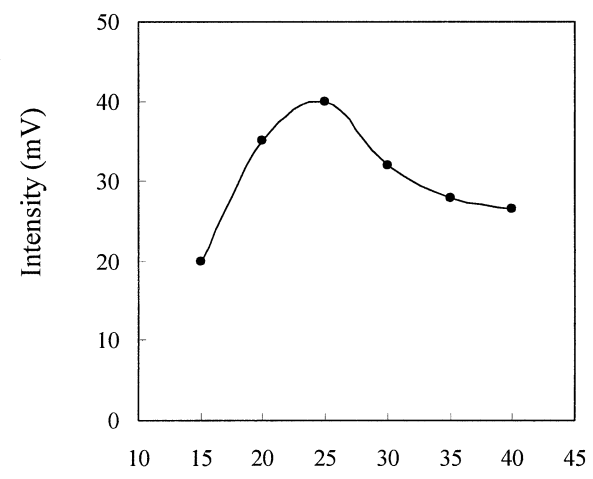

Extraction coil diameter ( $\mathrm{mm})$

Fig. 5 Emission signal as a function of the extraction coil diameter, concentration of $400 \mu \mathrm{g} / \mathrm{l}$ ytterbium.

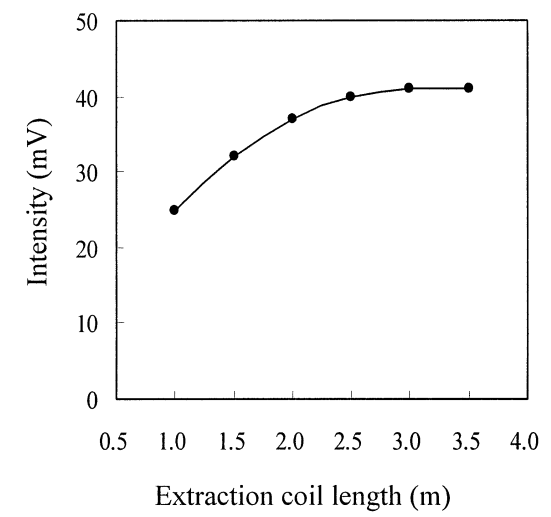

Fig. 6 Emission signal as a function of the extraction coil length, concentration of $400 \mu \mathrm{g} / \mathrm{l}$ ytterbium.

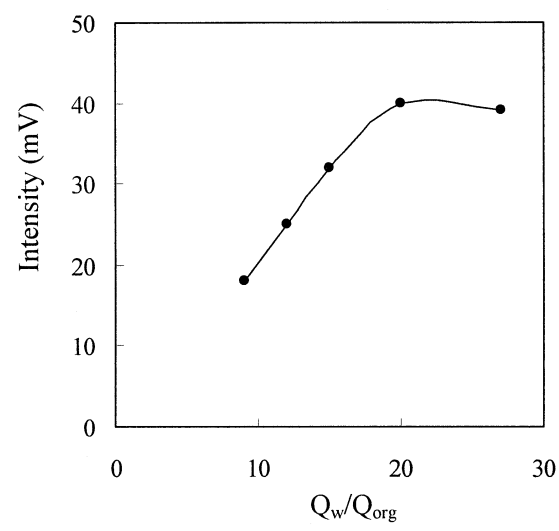

Fig. 7 Emission signal as a function of $Q_{\mathrm{w}} / Q_{\text {org }}$, varying $Q_{\mathrm{w}}$ at fixed $Q_{\text {org. }} Q_{\text {org }}=0.20 \mathrm{ml} / \mathrm{min}$, concentration of $400 \mu \mathrm{g} / \mathrm{l}$ ytterbium.

materials (which have different concentration ranges and different matrix compositions) are in good agreement with the certified values and the precision is acceptable.

\section{Conclusions}

In solvent extraction for ICP-AES, a stable plasma discharge with organic-solvent loading will be obtained if careful 


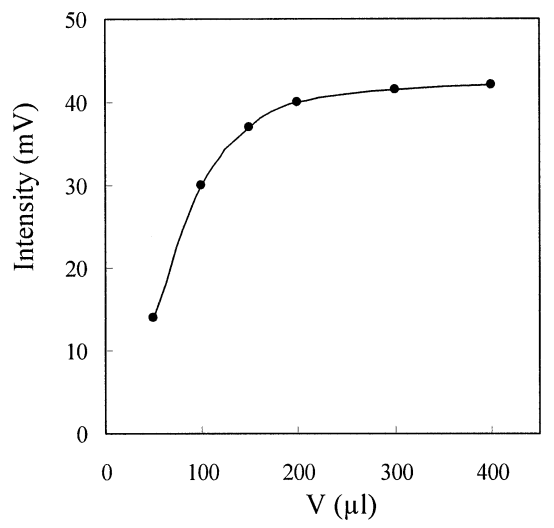

Fig. 8 Emission signal as a function of the injected volume of the solvent extract, concentration of $400 \mu \mathrm{g} / \mathrm{l}$ ytterbium.

optimization of the ICP operating parameters and solvent plasma loading are done. Under the optimized operating conditions, the plasma discharge is robust, which permits complete atomization of the sample and the excitation and ionization of free atoms.

The combination of FI-LLE and ICP-AES is an effective way to improve the detection sensitivity, and good analytical performance can be achieved. The proposed systems of FI-LLE preconcentration associated with ICP-AES allow for simultaneous multi-element determinations in geological samples with good accuracy and good reproducibility. It is clear that, in order to expand the applications of the combined FI-LLE and ICP-AES techniques, different organic solvents must be further investigated.

\section{Acknowledgements}

This work was supported financially by the Chinese Academy of Sciences (Innovation Project No. KZCX2-105).

\section{References}

1. X. Li, B. J. Coles, M. H. Ramsey, and I. Thornton, Chem. Geol., 1995, 124, 109.

2. S. L. Lin and C. Peng, J. Anal. At. Spectrom., 1990, 5, 509.

3. R. K. Tepe, T. Jacksier, and R. M. Barnes, J. Anal. At. Spectrom., 1998, 13, 989.

4. S. Farias and P. Smichowski, J. Anal. At. Spectrom., 1999, $14,809$.

5. E. Vassileva and M. Hoenig, Spectrochim. Acta Part B, 2001, 56, 223.

6. M. Hoenig, H. Docekalova, and H. Baeten, J. Anal. At. Spectrom., 1998, 13, 195.

7. N. Daskalova and Iv. Boevski, Spectrochim. Acta Part B, 1999, 54, 1099.

8. M. Villaneuva, M. Catasus, E. D. Salin, and M. Pomares, $J$. Anal. At. Spectrom., 2000, 15, 877.

9. Z. Horvath, A. Lasztity, and R. M. Barnes, Spectrochim. Acta Rev., 1991, 14, 45.

10. A. W. Boorn and R. F. Browner, Anal. Chem., 1982, 54, 1402.

11. P. W. J. M. Boumans and M. Ch. Lux-Steiner, Spectrochim. Acta Part B, 1982, 37, 97.

12. F. J. M. J. Maessen, G. Kreuning, and J. Balke, Spectrochim. Acta Part B, 1986, 41, 3.
Table 4 Comparison of the analytical performance between direct nebulization with aqueous solution and FI-LLE-ICP-AES

\begin{tabular}{|c|c|c|c|c|}
\hline \multirow{2}{*}{ Element } & \multicolumn{2}{|c|}{ Aqueous solution } & \multicolumn{2}{|c|}{ FI-LLE } \\
\hline & $\begin{array}{c}\mathrm{DL}(3 \sigma) \\
(\mathrm{ng} / \mathrm{ml})\end{array}$ & $\begin{array}{l}\text { Precision }^{\mathrm{a}} \\
\text { (\% RSD) }\end{array}$ & $\begin{array}{c}\mathrm{DL}(3 \sigma) \\
(\mathrm{ng} / \mathrm{ml})\end{array}$ & $\begin{array}{l}\text { Precision }^{b} \\
\text { (\% RSD) }\end{array}$ \\
\hline La 333.75 & 840 & 5.6 & 15 & 4.6 \\
\hline Nd 309.42 & 560 & 7.4 & 9 & 5.2 \\
\hline Sm 359.26 & 400 & 3.1 & 6.2 & 2.8 \\
\hline Eu 381.97 & 270 & 3.3 & 3.8 & 2.1 \\
\hline Er 337.3 & 200 & 3.6 & 3.2 & 1.8 \\
\hline Yb 328.94 & 200 & 3.4 & 2.8 & 4.7 \\
\hline Y 324.23 & 120 & 1.6 & 1.8 & 2.4 \\
\hline \multicolumn{3}{|c|}{ Loading time (s) } & \multicolumn{2}{|c|}{40} \\
\hline \multicolumn{3}{|c|}{ Sample throughout (samples/h) } & \multicolumn{2}{|c|}{27} \\
\hline \multicolumn{3}{|c|}{ Sample consumption (ml) } & \multicolumn{2}{|c|}{4.0} \\
\hline \multicolumn{3}{|c|}{ TBP consumption (ml) } & \multicolumn{2}{|c|}{0.20} \\
\hline
\end{tabular}

a. Relative standard deviation (\%), $n=9$.

b. Relative standard deviation $(\%), n=7$.

Table 5 FI-LLE-ICP-AES analysis of standard reference materials $(\mu \mathrm{g} / \mathrm{g})$

\begin{tabular}{|c|c|c|c|c|}
\hline \multirow{2}{*}{ Element } & \multicolumn{2}{|c|}{ GBW07234 } & \multicolumn{2}{|c|}{ GBW07236 } \\
\hline & Certified $^{a}$ & Found $^{b}$ & Certified $^{\mathrm{a}}$ & Found $^{\mathrm{b}}$ \\
\hline $\mathrm{La}$ & $40.3 \pm 2.7$ & $38.2 \pm 1.5$ & $31.2 \pm 1.5$ & $30.0 \pm 2.0$ \\
\hline $\mathrm{Nd}$ & $29.4 \pm 2.6$ & $26.8 \pm 1.8$ & $23.4 \pm 1.2$ & $21.6 \pm 1.6$ \\
\hline $\mathrm{Sm}$ & $5.1 \pm 0.4$ & $4.8 \pm 0.5$ & $4.6 \pm 0.3$ & $4.2 \pm 0.2$ \\
\hline $\mathrm{Eu}$ & $1.3 \pm 0.1$ & $1.5 \pm 0.1$ & $0.82 \pm 0.07$ & $0.88 \pm 0.1$ \\
\hline Er & $1.3 \pm 0.2$ & $1.4 \pm 0.1$ & $1.6 \pm 0.3$ & $1.5 \pm 0.1$ \\
\hline $\mathrm{Yb}$ & $1.2 \pm 0.1$ & $1.1 \pm 0.1$ & $1.7 \pm 0.3$ & $1.8 \pm 0.2$ \\
\hline $\mathrm{Y}$ & $11.8 \pm 0.9$ & $11.5 \pm 1.0$ & $16.2 \pm 2.1$ & $17.0 \pm 1.8$ \\
\hline
\end{tabular}

The standard reference materials are the national standard reference sample of rocks (China) obtained from China University of Geosciences.

a. Mean and standard deviation for no less than 15 replicates.

b. Mean and standard deviation for three replicates.

13. C. K. Pan, G. X. Zhu, and R. F. Browner, J. Anal. At Spectrom., 1990, 5, 537 .

14. D. G. Weir and M. W. Blades, J. Anal. At. Spectrom., 1994, 9, 1311.

15. D. G. Weir and M. W. Blades, J. Anal. At. Spectrom., 1994, 9, 1323.

16. J. Ruzicka and E. H. Hansen, "Flow Injection Analysis", 2nd ed., 1988, Wiley, New York.

17. B. Kalberg and G. E. Pacey, "Flow Injection Analysis (A Practical Guide)", 1989, Elsevier, Amsterdam.

18. G. Tao and Z. Fang, Spectrochim. Acta Part B, 1995, 50, 1747.

19. M. D. Luque de Castro and M. Valcárcel, "Flow injection analysis", in "Automation in the Laboratory", ed. A. J. Hurst, 1995, VCH Publishers, New York.

20. M. Valcárcel and M. Gallego, "Flow-injection on-line liquid-liquid separation and preconcentration atomic spectrometry", in "Flow Analysis with Atomic Spectrometric Detectors", ed. A. Sanz-Medel, 1999, Elsevier, Amsterdam.

21. S. L. Lin and H. P. Hwang, Talanta, 1993, 40, 1077.

22. S. L. Lin, Q. Shui, H. Qiu, and Z. Tang, Spectrochim. Acta Part B, 1996, 51, 1769.

23. J. M. Mermet, Anal. Chim. Acta, 1991, 250, 85. 